\title{
HIF-1a, HIF-2a, and ProExC: diagnostic or prognostic relevance in conjunctival intraepithelial neoplasia?
}

\author{
Simone Nuessle ${ }^{1}$ (D) Daniel Soriano ${ }^{1} \cdot$ Daniel Boehringer $^{1} \cdot$ Hans Mittelviefhaus $^{1} \cdot$ Clemens Lange $^{1}$. \\ Thomas Reinhard $^{1}$ • Lisa Atzrodt ${ }^{1}$ • Claudia Auw-Haedrich ${ }^{1}$
}

Received: 1 December 2019 / Revised: 26 April 2020 / Accepted: 6 May 2020 / Published online: 26 May 2020

(C) The Author(s) 2020

\begin{abstract}
Purpose The aim of this study was to investigate HIF-1 $\alpha$, HIF-2 $\alpha$, and ProExC expression in conjunctival intraepithelial neoplasia $(\mathrm{CIN})$, to differentiate between metaplasia and dysplasia, and to access their value as diagnostic and prognostic immunohistochemical markers. Recurrence and progression into SCC (squamous cell carcinoma) were defined as endpoints.

Methods Forty-three specimens including CIN I (2), CIN II (9), CIN III (29), with and without metaplasia, and metaplasia alone (3), as well as 21 conjunctival control specimens, were stained with antibodies against HIF-1 $\alpha$, HIF-2 $\alpha$, and ProExC. The percentage of positively stained cells were calculated and used for further analysis.

Results The mean percentages of HIF-1 $\alpha$ and HIF- $2 \alpha$ were not increased in CIN. In comparison, the expressions of these markers were even significantly elevated in control specimens $(p<0.001)$. Upper epithelial cells in CIN were more often ProExC-positive compared with normal conjunctiva or metaplasia ( $p=0.06$ and $p=0.07$ ). Cox proportional-hazards analysis was performed for characterization of factors influencing the combined endpoint and showed a significant elevated hazard ratio for staining with ProExC ( $p=0.04)$ compared with HIF-1 $\alpha(p=0.26)$ and HIF-2 $\alpha(p=0.49)$.

Conclusion Our study shows that HIF-1 $\alpha$ and HIF-2 $\alpha$ do not serve as diagnostic or prognostic markers in CIN. ProExC seems to be a potential indicator for CIN, but not a reliable diagnostic marker. However, control specimens occasionally also display a high percentage of ProExC-positive cells and staining over the entire epithelial layer.
\end{abstract}

Keywords Conjunctival intraepithelial neoplasia $\cdot \mathrm{HIF} \cdot$ ProExC $\cdot$ immunohistochemistry

\section{Introduction}

Conjunctival intraepithelial neoplasia (CIN) and squamous cell carcinoma (SCC) are rare tumors but are among the most common conjunctival malignancies with an incidence of SCC between $0.03 / 100000$ persons per year in the USA and 3.4 and 3.0 cases/year/100000 in Zimbabwe $[1,2]$.

CIN can present as a simple dysplasia to carcinoma in situ. The diagnosis of dysplasia and the grading of CIN are made histopathologically by means of subjective assessment. Classification and grading parallel cervical intraepithelial neoplasia (cervical IN). CIN grade depends on the proportional

Simone Nuessle

simone.nuessle@uniklinik-freiburg.de

1 Eye Center, Medical Center, Faculty of Medicine, University of Freiburg, Killianstr. 5, 79106 Freiburg im Breisgau, Germany distribution of dysplasia in relation to the epithelial height [3]. By analogy to the cervical IN, there are three different grades which range from CIN grade I with dysplasia of the basal third to CIN grade III or carcinoma in situ with dysplasia over $2 / 3$ of the whole thickness of the epithelium [4].

Dysplasia is defined by morphological criteria such as atypical, enlarged, and/or hyperchromatic nuclei; increased mitosis; and apoptosis. These criteria are in some extent quite subjective. Therefore, additional immunohistochemical stains supporting malignancy would be helpful, also in differentiating those tumors consisting of metaplasia alone from those with $\mathrm{CIN}$ and additional metaplasia.

In cervical IN, the markers HIF- $1 \alpha$ and ProExC have already been evaluated as diagnostic markers [5-7].

A higher expression of HIF (hypoxia-inducible factors) was linked to poorer prognosis in a large number of different tumors [7-9]. HIFs, mainly HIF-1 $\alpha$ and HIF-2 $\alpha$, are heterodimeric transcription factors which are rapidly degraded under normoxic conditions by hydroxylation (PHD 1-3), binding to 
the VHL (von Hippel Lindau) protein and together marked by E3-ubiquitin-ligase complex before proteasomal degradation (Fig. 1). HIF is known to have a key role in cellular responses to hypoxia and is an important regulator of tumorigenesis including proliferation, differentiation, angiogenesis, immortalization, metastasis, and apoptosis [10-13].

In cervical $\mathrm{IN}$, studies observed a higher expression of HIF- $1 \alpha$ in high-grade cervical IN compared with that in normal cervical epithelium $[5,7]$. Furthermore, No et al. also showed higher expression of HIF- $1 \alpha$ in high-grade in comparison with low-grade dysplasia.

ProExC consists of two monoclonal antibodies against TOP2A (topoisomerase $2 \alpha$ ) and MCM2 (minichromosome maintenance protein 2) which are two important factors in DNA replication $[14,15]$. ProExC was shown to indicate a correlation between expression and cervical IN grades with significant higher expression of higher cervical IN grades whereas specimens with metaplasia showed less ProExC expression [6].

Due to the similarity between cervical and conjunctival intraepithelial neoplasia, we focused on the markers HIF-1 $\alpha$ and ProExC as potential diagnostic and prognostic markers of CIN. Besides this, we aimed to investigate HIF- $2 \alpha$ in CIN which level of expression has been found increased in various tumors like bladder, breast, colon, and also cervical carcinomas [9].
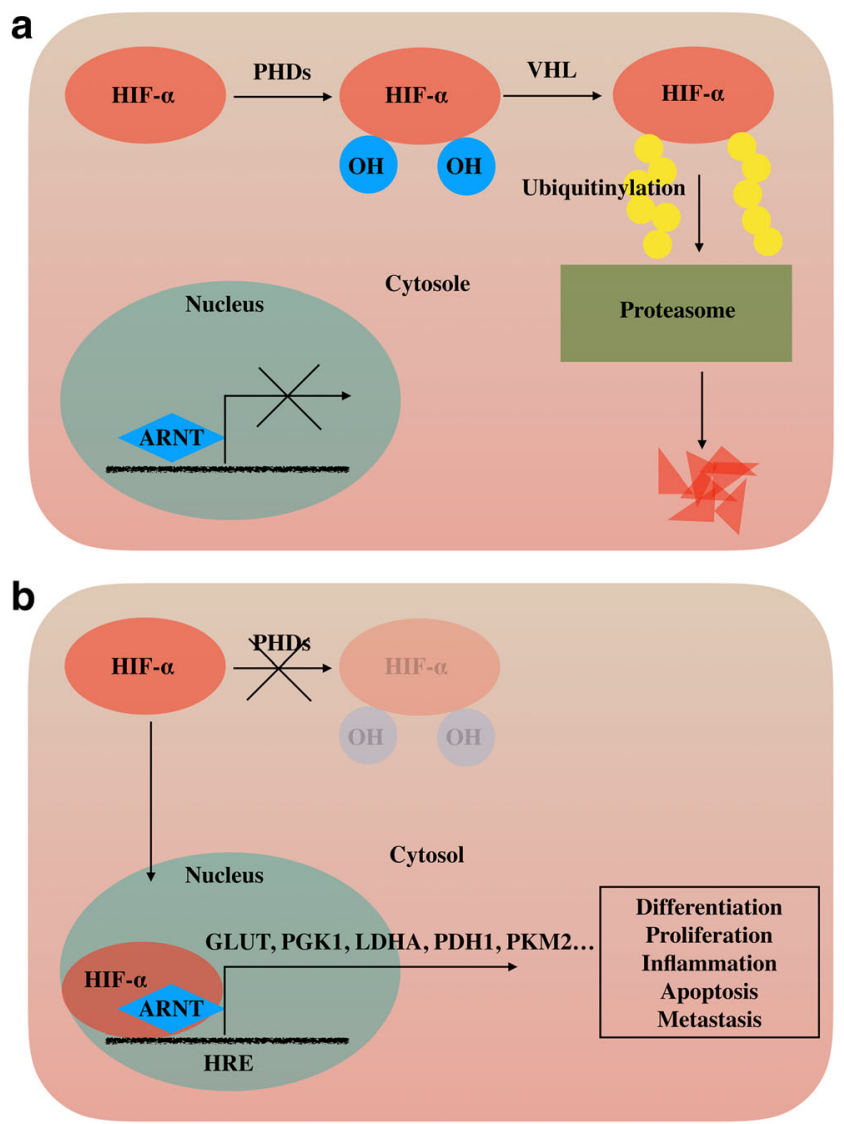

Fig. 1 Schematic representation of the oxygen-dependent degradation of HIF- $\alpha$ by ubiquitination (modified from Rankin and Giaccia [9])

\section{Material and methods}

The study was approved by the ethics committee of the Albert-Ludwig University Freiburg, Germany; written informed consent was obtained from the study participants. Forty-three conjunctival specimens with diagnosed CIN or metaplasia (details in results) which had been excised at the Eye Center Freiburg between 2004 and 2013 were included. Control specimens originated from excess conjunctiva that had been resected at the end of buckle retinal detachment surgeries.

\section{Preparation of the specimen}

After excision, the specimens were fixed in $4 \%$ formaldehyde in phosphate buffer of $7.0 \mathrm{pH}$ for $24-48 \mathrm{~h}$, dehydrated in increasing concentrations of ethanol (70-100\%) and xylol and infiltrated with paraffin (TP 1020, Leica, Wetzlar, Germany). Sections of $4 \mu \mathrm{m}$ thickness were cut and floated on deionized water at $20^{\circ} \mathrm{C}$, and single sections were mounted on X-tra adhesive glass slides (Leica, Wetzlar, Germany). Slides were stretched in a water bath at $47-48^{\circ} \mathrm{C}$, subsequently dried at $60{ }^{\circ} \mathrm{C}$ for $30 \mathrm{~min}$, and stored at a room temperature in darkness.

Prior to immunohistochemical staining, the specimens were deparaffinized and rehydrated by xylol and in decreasing concentrations of ethanol (100-70\%). Afterward, they were demasked using a citrate buffer of $\mathrm{pH} 6$, heated in deionized water at $95{ }^{\circ} \mathrm{C}$ for $20 \mathrm{~min}$, and slowly cooled down for $20 \mathrm{~min}$. Now, they were stained immunohistochemically using the catalyzed reporter deposition method by biotin-free tyramide amplification with the DAKO CSA II kit and washed with $0.05 \mathrm{M}$ TBST-wash buffer between the steps as recently described [16]. First, the endogen peroxidase was blocked by $3 \%$ hydrogen peroxide for $15 \mathrm{~min}$ and the slides were incubated in $0.015 \mathrm{M}$ sodium azide buffer before $100 \mu \mathrm{l}$ of the diluted mouse monoclonal antibodies (pre-diluted ProExC, HIF- $1 \propto 1: 8000$ and HIF-2 $\alpha$ 1:500) were applied for $15 \mathrm{~min}$ at a room temperature and $18-21 \mathrm{~h}$ at $4{ }^{\circ} \mathrm{C}$. Applied were the secondary antibody from the DAKO CSA II kit for $60 \mathrm{~min}$, the fluorescein-tyramide and hydrogen peroxide for amplification for $15 \mathrm{~min}$ in the dark, the tertiary antibody from the DAKO CSA II kit for $15 \mathrm{~min}$, and chromogen AEC under microscopical control of the positive control $4 \mathrm{~min}$ for ProExC and $10 \mathrm{~min}$ for HIF before counterstaining with Harris hematoxylin for $2 \mathrm{~s}$.

\section{Evaluation of the specimens}

Two to five representative sections of each specimen were photographed at a magnification of $\times 400$ (HIF) and $\times 200$ (ProExC), respectively. The cell number was determined by a self-programmed, validated, automated, cell counting 
software (reliability of $100 \%$ ). Positive nuclear staining for HIF- $1 \alpha$ and HIF- $2 \alpha$, and a strong cytoplasmic staining for HIF- $2 \alpha$ were determined by counting manually, whereas positively stained cells for ProExC were counted automatically by the computer-based calculator. The percentage of positively stained cells for HIF- $1 \alpha$, HIF- $2 \alpha$, and ProExC was calculated. The results were subdivided into three groups: strongly (> $1.10 \%(\mathrm{HIF}-1 \alpha),>9.38 \%(\mathrm{HIF}-2 \alpha)$, and $>59.19 \%$ (ProExC) positively stained cells), medium $(0.43-1.10 \%$ (HIF-1 $\alpha$ ), $1.27-9.38 \%$ (HIF-2 $\alpha$ ), and $43.5-59.19 \%$ (ProExC) positively stained cells), and weakly $(<0.43 \%$ $($ HIF- $1 \alpha),<1.27 \%$ (HIF- $2 \alpha$ ), and $<43.5 \%$ (ProExC) positively stained cells) expressed. In addition, the distribution of positively stained cells for ProExC regarding the vertical extension of epithelial thickness was determined and divided into basal, middle, and upper third.

To investigate whether inflammation might have an impact on the immunoreaction, we also counted manually all intraepithelial as well as subepithelial inflammatory cells (lymphocytes) in each photograph. Four groups were defined as 0 : no inflammatory cell, $1:<5$ inflammatory cells, $2: 5-20$ inflammatory cells, and 3: $>20$ inflammatory cells.

We also reviewed the medical records for the following: age at time of surgery, sex, histopathological diagnosis, and days until tumor-associated events (recurrence and SCC development, which were combined for further analysis).

We fitted a proportional hazards Cox regression model in order to assess the influence of the aforementioned covariates on tumor-related events. Significant level was set at $p$ value $<$ 0.05 .

\section{Results}

\section{Baseline data}

The study cohort comprised 12 women and 31 men with a median age of 63 years (27-87 years) at time of surgery. Of these 43 included specimens, 3 were diagnosed with metaplasia alone, 8 with CIN without metaplasia, and 32 with CIN and metaplasia. Referring to the CIN specimen, 2 were classified as CIN I, 9 as CIN II, and 29 as CIN III. The median follow-up period was 1013 days (interquartile range, 4862075 days). None of the included patients developed metastases or died in association with the included disease. Recurrence was shown in 9 patients (23\%) after a median time of 301 days (range, 110-2075 days), most of them within the first 2 years. One was initially diagnosed with CIN I, 2 with CIN II, and 6 with CIN III. Three patients (7\%) developed SCC after 441, 806, and 2653 days. All of them were male patients with initial diagnosis of CIN III and concomitant autoimmune dermatitis. In metaplasia, there was-as expected - no tumor-associated event. The control group comprised
21 normal conjunctival specimens from 9 female and 12 male patients with a median age of 59.5 years (33-82 years) (Fig. 2 and Table 1).

\section{Percentage HIF-1 $a$, HIF-2 $a$, and ProExC expression}

The mean percentages of HIF- $1 \alpha$-positive cells in all CIN specimens with or without metaplasia were $10.9 \% \pm 3.0 \%$ and $3.9 \% \pm 8.5 \%$, respectively. Notably, HIF- $1 \alpha$ expression was significantly elevated in normal conjunctival specimens compared with CIN without or with metaplasia with a mean percentage of $59.5 \% \pm 20.0 \%$ HIF- $1 \alpha$-positive cells $(p<$ 0.001) (Fig. 3c).

The mean proportion of HIF- $2 \alpha$ expression in all CIN specimens with or without metaplasia was calculated at $11.0 \% \pm 19.5 \%$ and $8.4 \% \pm 10.2 \%$, respectively. As already shown in HIF- $1 \alpha$ expression, the HIF- $2 \alpha$ expression as well was significantly elevated in the control group compared with CIN specimen with a mean percentage of $20.1 \% \pm 7.5 \%(p<$ 0.001) (Fig. 4c).

The proportion of ProExC-positive cells in CIN was similar to the control group with a mean percentage of $50.6 \% \pm$ $23.1 \%$ in CIN without metaplasia, $50.2 \% \pm 20.9 \%$ in CIN with metaplasia, and $45.2 \% \pm 17.3 \%$ in control specimens $(p$ $=0.55$ ).

Our analysis showed that there was no correlation between extent of inflammation and immunostaining $\left(R^{2}<0.04\right)$.

\section{Vertical extension of ProExC}

With regard to vertical extension of ProExC, we observed a correlation between the classification and the positively stained epithelial height, however missing statistical

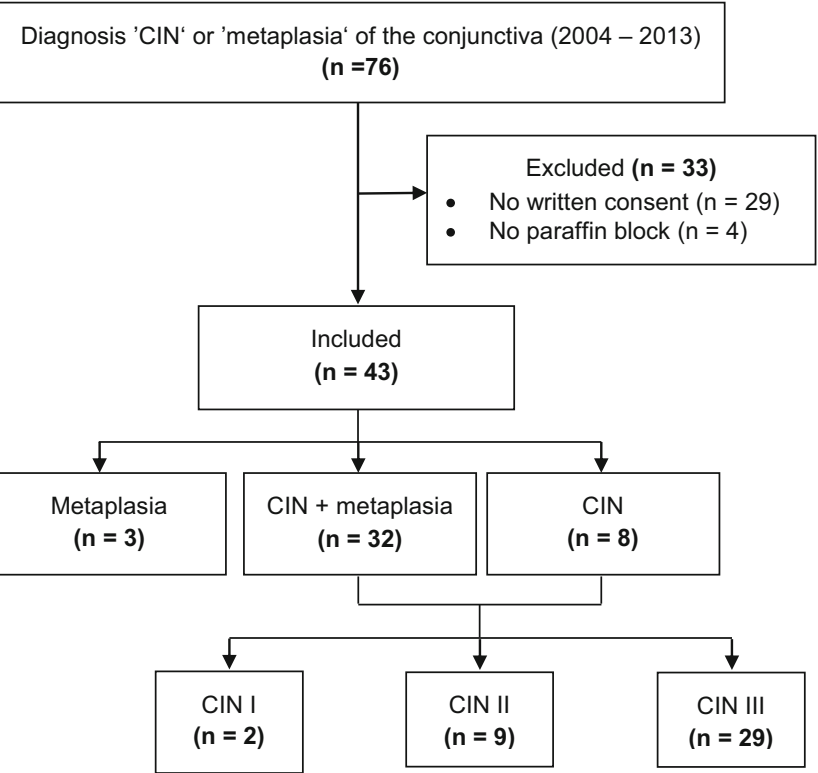

Fig. 2 Flow chart of baseline data 
Table 1 Baseline data of the 43 cases included in the study

\begin{tabular}{|c|c|c|c|c|c|c|}
\hline No. & Age (years) & Sex & Histological diagnosis & Follow-up time (days) & Recurrence of CIN & Progression to SCC \\
\hline 1 & 66.6 & $\mathrm{f}$ & Metaplasia & 3808 & No & No \\
\hline 2 & 52.7 & $\mathrm{~m}$ & Metaplasia & 3332 & No & No \\
\hline 3 & 63.0 & $\mathrm{~m}$ & Metaplasia & 2692 & No & No \\
\hline 4 & 52.0 & $\mathrm{~m}$ & CIN III + metaplasia & 2653 & Yes & Yes \\
\hline 5 & 54.1 & $\mathrm{~m}$ & CIN III & 441 & Yes & Yes \\
\hline 6 & 51.2 & $\mathrm{~m}$ & CIN III + metaplasia & 806 & No & Yes \\
\hline 7 & 70.2 & $\mathrm{~m}$ & CIN III + metaplasia & 1789 & Yes & No \\
\hline 8 & 63.40 & $\mathrm{~m}$ & CIN II & 859 & Yes & No \\
\hline 9 & 49.1 & $\mathrm{~m}$ & CIN III + metaplasia & 255 & Yes & No \\
\hline 10 & 37.0 & $\mathrm{f}$ & CIN I + metaplasia & 209 & Yes & No \\
\hline 11 & 48.2 & $\mathrm{~m}$ & CIN III + metaplasia & 2075 & Yes & No \\
\hline 12 & 46.4 & $\mathrm{~m}$ & CIN II + metaplasia & 279 & Yes & No \\
\hline 13 & 71.3 & $\mathrm{~m}$ & CIN III & 789 & Yes & No \\
\hline 14 & 65.0 & $\mathrm{~m}$ & CIN III + metaplasia & 4465 & No & No \\
\hline 15 & 76.6 & $\mathrm{f}$ & CIN III + metaplasia & 1526 & No & No \\
\hline 16 & 62.6 & $\mathrm{~m}$ & CIN II + metaplasia & 2555 & No & No \\
\hline 17 & 70.8 & $\mathrm{~m}$ & CIN III + metaplasia & 967 & No & No \\
\hline 18 & 46.5 & $\mathrm{f}$ & CIN III + metaplasia & 1383 & No & No \\
\hline 19 & 69.4 & $\mathrm{~m}$ & CIN III + metaplasia & 0 & No & No \\
\hline 20 & 87.1 & $\mathrm{~m}$ & CIN III + metaplasia & 3109 & No & No \\
\hline 21 & 66.4 & $\mathrm{f}$ & CIN I + metaplasia & 3040 & No & No \\
\hline 22 & 54.5 & $\mathrm{~m}$ & CIN III + metaplasia & 1561 & No & No \\
\hline 23 & 70.2 & $\mathrm{~m}$ & CIN III + metaplasia & 258 & No & No \\
\hline 24 & 78.4 & $\mathrm{~m}$ & CIN III & 774 & No & No \\
\hline 25 & 55.2 & $\mathrm{~m}$ & CIN III + metaplasia & 803 & No & No \\
\hline 26 & 67.4 & $\mathrm{f}$ & CIN II & 2438 & No & No \\
\hline 27 & 42.9 & $\mathrm{~m}$ & CIN III & 2617 & No & No \\
\hline 28 & 27.9 & $\mathrm{~m}$ & CIN II + metaplasia & 851 & No & No \\
\hline 29 & 77.6 & $\mathrm{f}$ & CIN III + metaplasia & 1883 & No & No \\
\hline 30 & 82.4 & $\mathrm{~m}$ & CIN III + metaplasia & 218 & No & No \\
\hline 31 & 38.9 & $\mathrm{f}$ & CIN III + metaplasia & 1342 & No & No \\
\hline 32 & 29.2 & $\mathrm{f}$ & CIN III & 1502 & No & No \\
\hline 33 & 72.5 & $\mathrm{~m}$ & CIN III + metaplasia & 1138 & No & No \\
\hline 34 & 63.0 & $\mathrm{f}$ & CIN III + metaplasia & 798 & No & No \\
\hline 35 & 67.4 & $\mathrm{~m}$ & CIN II + metaplasia & 486 & No & No \\
\hline 36 & 73.7 & $\mathrm{~m}$ & CIN II + metaplasia & 1557 & No & No \\
\hline 37 & 62.4 & $\mathrm{f}$ & CIN III + metaplasia & 978 & No & No \\
\hline 38 & 68.0 & $\mathrm{~m}$ & CIN III + metaplasia & 2 & No & No \\
\hline 39 & 62.3 & $\mathrm{~m}$ & CIN II & 1013 & No & No \\
\hline 40 & 43.3 & $\mathrm{~m}$ & CIN III + metaplasia & 293 & No & No \\
\hline 41 & 82.1 & $\mathrm{~m}$ & CIN III + metaplasia & 1258 & No & No \\
\hline 42 & 65.1 & $\mathrm{~m}$ & CIN III + metaplasia & 1 & No & No \\
\hline 43 & 63.4 & $\mathrm{f}$ & CIN II + metaplasia & 811 & No & No \\
\hline
\end{tabular}

significance. About $52 \%$ of the normal conjunctival specimens showed positive staining for ProExC particularly in the basal third of the epithelium. In about $60 \%$ of the specimens with CIN, in contrast, cells in the upper third of the epithelium expressed ProExC $(p=0.06)$.

Moreover, we observed a statistically significant correlation between the percentage expression of ProExC and the level of stained epithelial thickness $(p<0.001)$ (Fig. 5e).

\section{HIF and ProExC expression and tumor-related events}

No statistically significant difference of HIF- $1 \alpha$ and HIF- $2 \alpha$ expression was found between CIN specimen developing recurrence or progressing to $\mathrm{SCC}$ compared with $\mathrm{CIN}$ without any of these tumor-related events. Notably, the mean expression of HIF- $1 \alpha$ in specimens with CIN recurrence and progression to SCC were even lower (Table 2).

In the Cox regression, we observed a higher ProExC expression in specimens with CIN recurrence $(p=0.12)$ and progression to SCC $(p=0.19)$ compared with those without any tumor-related event, but without statistical significance. Moreover, all three specimens with progression to SCC during the observation period were stained up to the upper third of the epithelium. Nevertheless, the difference of those cases without progression to SCC was not statistically significant $(p=0.34)$.

However, the Cox proportional hazards showed a significant elevated hazard ratio for staining with ProExC compared with HIF (Table 3 and Fig. 6). 
Fig. 3 HIF-1 $\alpha: \mathbf{a}+\mathbf{b}$

Representative

immunohistochemical nuclear staining (red) for HIF-1 $\alpha$ (scale bar $=100 \mu \mathrm{m})$. a Nuclear staining in normal conjunctiva and $\mathbf{b}$ in CIN III with metaplasia. c Boxplot for percentage expression of HIF- $1 \alpha$ compared with controls, CIN, and CIN with metaplasia $(p<0.001)$ a

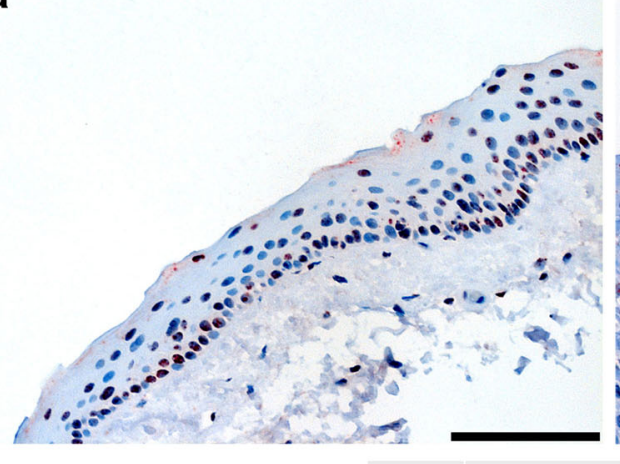

b

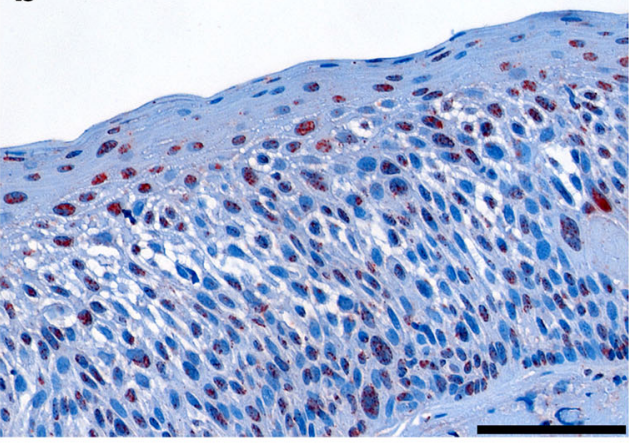

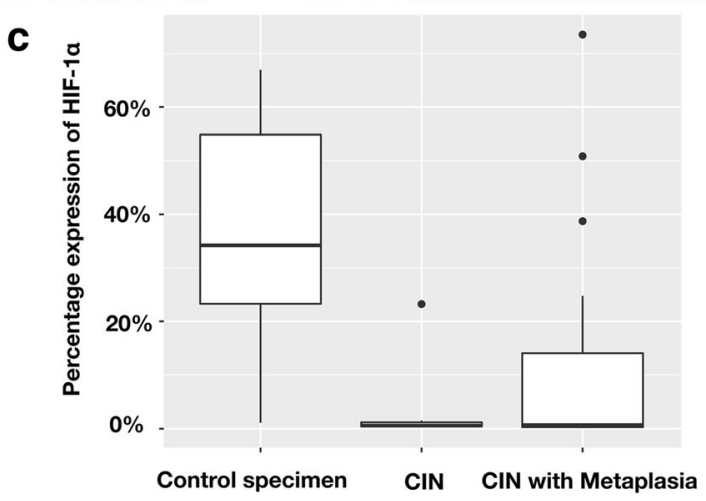

\section{Discussion}

HIF- $1 \alpha$ and HIF- $2 \alpha$ are known to drive gene expression that support metabolism under hypoxic conditions. The activation of HIF- $1 \alpha$ and HIF- $2 \alpha$ seems to be common in malignancies [9]. Talks et al. described an overexpression of HIF- $1 \alpha$ and HIF- $2 \alpha$ in breast, colon, ovarian, pancreatic, prostate, renal, and hepatocellular carcinomas [8]. Moreover, No et al. have
Fig. 4 HIF-2 $\alpha$ : a + b

Representative

immunohistochemical nuclear and cytoplasmic staining (red) for HIF- $2 \alpha$ (scale bar $=100 \mu \mathrm{m})$. a Nuclear staining in normal conjunctiva and $\mathbf{b}$ in CIN III with metaplasia. c Boxplot for percentage expression of HIF- $1 \alpha$ compared with controls, CIN, and CIN with metaplasia $(p<0.001)$ a
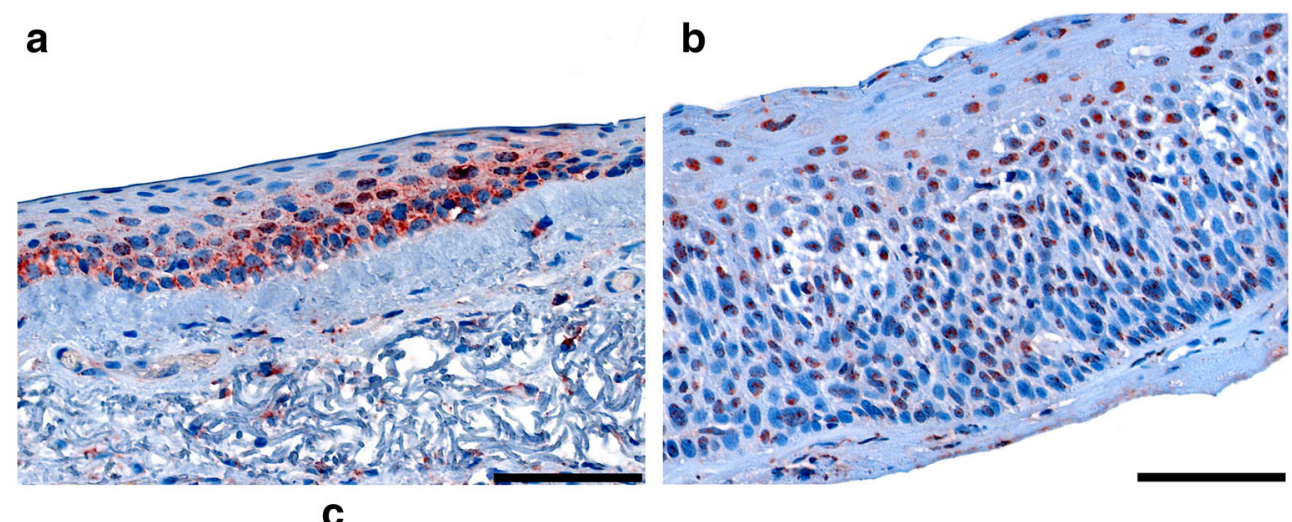

C

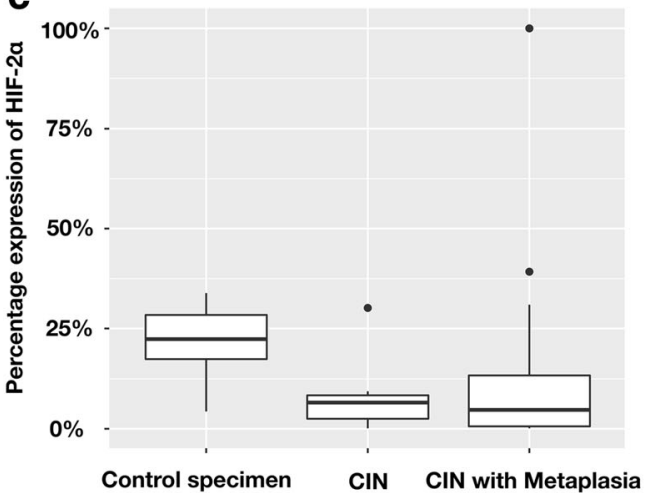




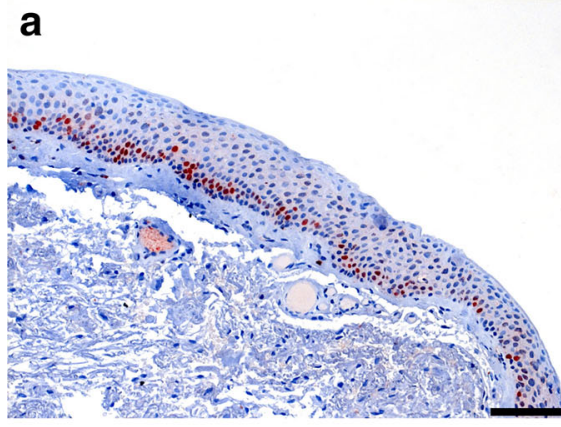

d

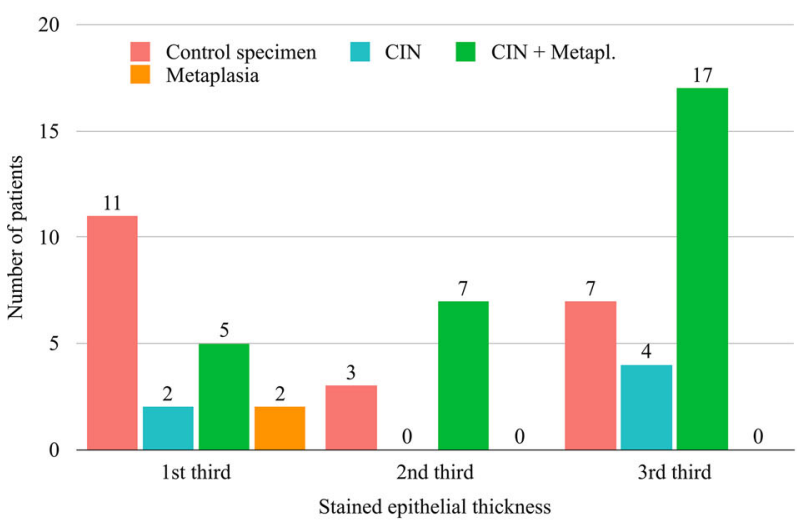

Fig. 5 ProExC: a-c Representative immunohistochemical nuclear staining (red) for ProExC in different vertical extension (magnification $\times 200$, scale bar $=100 \mu \mathrm{m}$ ). a Nuclear staining in the basal third of the epithelium in normal conjunctiva, $\mathbf{b}$ in the basal $2 / 3$ of the epithelium in CIN grade II, and $\mathbf{c}$ of the whole thickness of the epithelium in carcinoma

shown a notably increased expression of HIF-1 $\alpha$ at higher cervical IN grades [5] and Birner et al. observed that HIF- $1 \alpha$ was highly expressed in cervical cancer and in high-grade CIN compared with normal cervix [7]. Furthermore, clinical studies have revealed that higher expression of HIF-1 $\alpha$ and HIF- $2 \alpha$ seems to be associated with a poorer prognosis in many tumors [9]. In contrast, HIF is also able to reduce tumor growth by cell cycle arrest and control of proapoptotic genes $[17,18]$.

The present study showed low expression of HIF- $1 \alpha$ and HIF- $2 \alpha$ in CIN. The conjunctiva is well supplied with blood and has a high degree of external humidification and oxygen supply. In accordance to the cancer stem cell hypothesis, it is assumed that cells carrying the best situation-related mutation ("survival of the fittest") accumulate in tumors [19]. We assume that HIF is downregulated to avoid cell cycle arrest and HIF-induced apoptosis because all cells of the conjunctiva may be well oxygenated, even if dysplastic. Schoelles et al., different investigators from our eye center, also analyzed HIF$1 \alpha$ and HIF- $2 \alpha$ and the controls were treated in the same way as the specimens of our study. Their unpublished results include a higher expression of HIF- $1 \alpha$ and HIF- $2 \alpha$ in the controls compared with pterygium specimens. In contrast, we
C

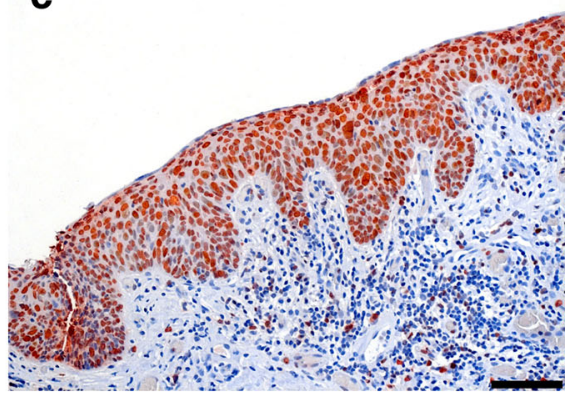

in situ. d Level of maximal (basal/middle/upper third $=1 \mathrm{st} / 2 \mathrm{nd} / 3 \mathrm{rd}$ ) stained epithelial thickness with ProExC. Comparison between the classifications. e Correlation between the percentage expression of ProExC and the stained epithelial thickness $(p<0.001)$

observed that the expressions of HIF- $1 \alpha$ and also of TKTL1 (transketolase-like protein 1), induced by hypoxia and HIF$1 \alpha$, were higher in SCC of the ocular adnexa (including the conjunctiva) in comparison with conjunctival papilloma. Higher HIF- $1 \alpha$ and TKTL-1 expression tended to be associated with a worse prognosis $[16,20,21]$. In the current study,

Table 2 Proportion of HIF- $1 \alpha$-, HIF- $2 \alpha$-, and ProExC-positive cells

\begin{tabular}{llrll}
\hline & $n$ & Mean & $p$ value \\
\hline HIF-1 $\alpha$ & CIN with recurrence & 8 & $3 \% \pm 6 \%$ & 0.75 \\
& CIN without recurrence & 30 & $10 \% \pm 18 \%$ & \\
& CIN with progression to SCC & 3 & $1 \% \pm 1 \%$ & 0.58 \\
& CIN without progression to SCC & 35 & $10 \% \pm 17 \%$ & \\
HIF-2 $\alpha$ & CIN with recurrence & 7 & $12 \% \pm 16 \%$ & 0.59 \\
& CIN without recurrence & 30 & $10 \% \pm 19 \%$ & \\
& CIN with progression to SCC & 3 & $4 \% \pm 3 \%$ & 0.55 \\
CIN without progression to SCC & 34 & $11 \% \pm 19 \%$ & \\
ProExC & 8 & $60 \% \pm 22 \%$ & 0.12 \\
& CIN with recurrence & 29 & $48 \% \pm 20 \%$ & \\
& CIN without recurrence & 3 & $70 \% \pm 29 \%$ & 0.19 \\
& CIN with progression to SCC & 34 & $49 \% \pm 20 \%$ & \\
\hline CIN without progression to SCC & & &
\end{tabular}


Table 3 Cox proportional hazards for characterization of significant factors influencing the combined endpoint

\begin{tabular}{lrr}
\hline Parameters & Hazard ratio & $p$ value \\
\hline HIF-1 $\alpha$ & 0.003 & 0.26 \\
HIF-2 $\alpha$ & 4.41 & 0.49 \\
ProExC & 79.91 & 0.04 \\
\hline
\end{tabular}

we only stained the initial CIN specimens but not the later CIN recurrences or SCC. In order to investigate these contradictory findings further, a longitudinal analysis of HIF or TKTL-1 expression is warranted.

The significantly increased expression of HIF in normal samples compared with CIN was unexpected, given the external oxygen supply. Our analysis hint towards intraoperative hypoxia in the controls $20-45 \mathrm{~min}$ by many severed capillaries before the control specimen was completely separated from the adjacent conjunctiva at the end of the surgery. The expression of HIF- $1 \alpha$ level of brain tissue increased significantly under experimental conditions in rats within less than $1 \mathrm{~h}$ after traumatic head injury [22]. For this reason, we speculate that the higher expression of HIF in the control specimens may be caused by a longer preexcisional hypoxia compared with the included CIN cases, which in contrast to the control specimens were immediately excised completely.

Furthermore, we investigated ProExC as a potential indicator for the classification of conjunctival specimens such as $\mathrm{CIN}$ and the course of the disease, but not as a reliable diagnostic marker. ProExC, a mix of two monoclonal antibodies against TOP2A and MCM2, has already been tested as a sufficient marker for proliferating cells and aberrant S-phase.

In our study, no significant difference in the proportion of ProExC-positive cells in CIN compared with normal conjunctival specimen was found $(p=0.55)$. This is in contrast to the findings of Guo et al. who investigated cervical specimens and showed a staining in over $50 \%$ of the dysplastic cells in highgrade cervical IN [6]. Nevertheless, our study revealed a tendency towards a more pronounced staining in specimens with recurrence or progression to SCC in the clinical course, but without statistical significance. This is in line with the results found in cervical IN and cervical carcinomas [6]. Regarding significance, it must be considered that a sample size consisting of three cases with progression to SCC is very small. Interestingly, all 3 patients progressing into SCC had autoimmune dermatitis (case 5 and 6 had atopic dermatitis and case 4 psoriasis) indicating smaller risk of patients without such an immune deviation to progress from CIN to SCC. However, compared with HIF- $1 \alpha$ and HIF- $2 \alpha$, there was a statistically significant higher hazard ratio for ProExC staining within the first 4 years for $\mathrm{CIN}$ to develop a recurrence or SCC $(p=0.04)$. Evaluation of the vertical extension of ProExC staining showed that upper epithelial cells were more frequently stained in CIN compared with normal conjunctiva or metaplasia, however missing statistical significance $(p=0.06$ and $p=0.07$ ). Furthermore, we observed a statistically significant correlation between the proportion of ProExC-positive tumor cells and the vertical extension $(p<0.001)$.

A major drawback of our study is the low sample size. Our mostly negative findings may be due to a statistical power issue. Other limitations are the retrospective nature and the monocentric setting. Therefore, any clinic-related bias cannot be ruled out. However, a prospective large multicentric study is hard to perform because the conditions are very rare.

In conclusion, neither HIF- $1 \alpha$ nor HIF- $2 \alpha$ seems to be suitable as a diagnostic or prognostic marker in CIN. ProExC may be of some value for predicting recurrences or progression to $\mathrm{SCC}$ when analyzing the proportional staining. These findings should be confirmed in patients from other eye centers with a larger sample size.

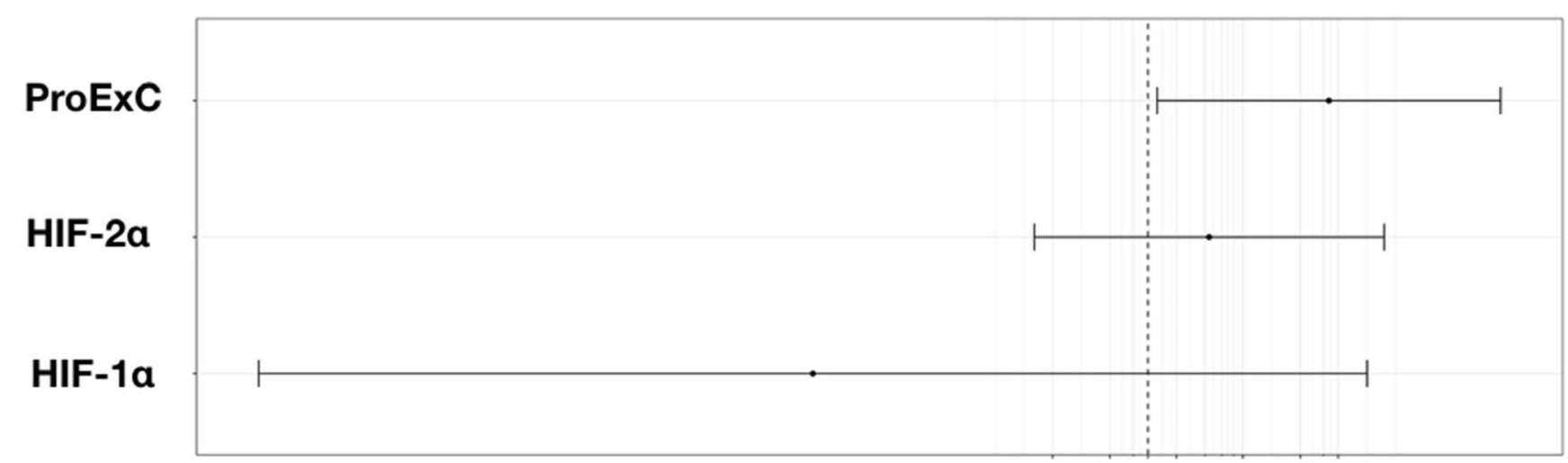

$0,10,412 \quad 10 \quad 40100$

\section{Odds-Ratio}

Fig. 6 Odds ratio for ProExC, HIF- $2 \alpha$, and HIF- $1 \alpha$ as influencing factors on tumor-related events. $X$-axis: odds ratio (OR), dots: Hazard ratio, horizontal line: $95 \%$ confidence interval 
Acknowledgments We thank Renate Buchen, Sylvia Zeitler, and Brigitte Joos for technical assistance.

Funding information Open Access funding provided by Projekt DEAL.

\section{Compliance with ethical standards}

Conflict of interest The authors declare that they have no conflict of interest.

Ethical approval All procedures performed in studies involving human participants were in accordance with the ethical standards of the institutional and/or national research committee and with the 1964 Helsinki declaration and its later amendments or comparable ethical standards.

Informed consent Informed consent was obtained from all individual participants included in the study.

Open Access This article is licensed under a Creative Commons Attribution 4.0 International License, which permits use, sharing, adaptation, distribution and reproduction in any medium or format, as long as you give appropriate credit to the original author(s) and the source, provide a link to the Creative Commons licence, and indicate if changes were made. The images or other third party material in this article are included in the article's Creative Commons licence, unless indicated otherwise in a credit line to the material. If material is not included in the article's Creative Commons licence and your intended use is not permitted by statutory regulation or exceeds the permitted use, you will need to obtain permission directly from the copyright holder. To view a copy of this licence, visit http://creativecommons.org/licenses/by/4.0/.

\section{References}

1. Sun EC, Fears TR, Goedert JJ (1997) Epidemiology of squamous cell conjunctival cancer. Cancer Epidemiol Biomark Prev 6:73-77

2. Curado MP, Edwards B, Shin HR et al (2007) Cancer incidence in five continents, Volume IX. Cancer incidence in five continents, Volume IX

3. Lee GA, Hirst LW (1995) Ocular surface squamous neoplasia. Surv Ophthalmol 39:429-450

4. Shields CL, Shields JA (2004) Tumors of the conjunctiva and cornea. Surv Ophthalmol 49:3-24

5. No JH, Jo H, Kim S-H et al (2009) Expression of vascular endothelial growth factor and hypoxia inducible factor- $1 \alpha$ in cervical neoplasia. Ann N Y Acad Sci 1171:105-110. https://doi.org/10. 1111/j.1749-6632.2009.04891.x

6. Guo M, Baruch AC, Silva EG et al (2011) Efficacy of p16 and ProExC immunostaining in the detection of high-grade cervical intraepithelial neoplasia and cervical carcinoma. Am J Clin Pathol 135:212-220. https://doi.org/10.1309/AJCP1LLX8QMDXHHO

7. Birner P, Schindl M, Obermair A et al (2000) Overexpression of hypoxia-inducible factor 1alpha is a marker for an unfavorable prognosis in early-stage invasive cervical cancer. Cancer Res 60: 4693-4696
8. Talks KL, Turley H, Gatter KC et al (2000) The expression and distribution of the hypoxia-inducible factors HIF-1alpha and HIF2alpha in normal human tissues, cancers, and tumor-associated macrophages. Am J Pathol 157:411-421. https://doi.org/10.1016/ s0002-9440(10)64554-3

9. Rankin EB, Giaccia AJ (2008) The role of hypoxia-inducible factors in tumorigenesis. Cell Death Differ 15:678-685. https://doi. org/10.1038/cdd.2008.21

10. Epstein AC, Gleadle JM, McNeill LA et al (2001) C. elegans EGL9 and mammalian homologs define a family of dioxygenases that regulate HIF by prolyl hydroxylation. Cell 107:43-54. https://doi. org/10.1016/s0092-8674(01)00507-4

11. Masson N, Willam C, Maxwell PH et al (2001) Independent function of two destruction domains in hypoxia-inducible factor-alpha chains activated by prolyl hydroxylation. EMBO J 20:5197-5206. https://doi.org/10.1093/emboj/20.18.5197

12. Maxwell PH, Wiesener MS, Chang GW et al (1999) The tumour suppressor protein VHL targets hypoxia-inducible factors for oxygen-dependent proteolysis. Nature 399:271-275. https://doi. org/10.1038/20459

13. Lange CAK, Bainbridge JWB (2012) Oxygen sensing in retinal health and disease. Ophthalmologica 227:115-131. https://doi. org/10.1159/000331418

14. Vos SM, Tretter EM, Schmidt BH, Berger JM (2011) All tangled up: how cells direct, manage and exploit topoisomerase function. Nat Rev Mol Cell Biol 12:827-841. https://doi.org/10.1038/ nrm 3228

15. Newlon CS (1997) Putting it all together: building a prereplicative complex. Cell 91:717-720. https://doi.org/10.1016/s00928674(00)80459-6

16. Lange CAK, Lehnert P, Boneva SK et al (2018) Increased expression of hypoxia-inducible factor- 1 alpha and its impact on transcriptional changes and prognosis in malignant tumours of the ocular adnexa. Eye (Lond) 32:1772-1782. https://doi.org/10.1038/ s41433-018-0172-6

17. Koshiji M, Kageyama Y, Pete EA et al (2004) HIF-1alpha induces cell cycle arrest by functionally counteracting Myc. EMBO J 23: 1949-1956. https://doi.org/10.1038/sj.emboj.7600196

18. Sowter HM, Ratcliffe PJ, Watson P et al (2001) HIF-1-dependent regulation of hypoxic induction of the cell death factors BNIP3 and NIX in human tumors. Cancer Res 61:6669-6673

19. Reya T, Morrison SJ, Clarke MF, Weissman IL (2001) Stem cells, cancer, and cancer stem cells. Nature 414:105-111. https://doi.org/ $10.1038 / 35102167$

20. Lange CA, Tisch-Rottensteiner J, Böhringer D et al (2012) Enhanced TKTL1 expression in malignant tumors of the ocular adnexa predicts clinical outcome. Ophthalmology 119:1924 1929. https://doi.org/10.1016/j.ophtha.2012.03.037

21. Heller S, Maurer GD, Wanka C et al (2018) Gene suppression of transketolase-like protein 1 (TKTL1) sensitizes glioma cells to hypoxia and ionizing radiation. Int J Mol Sci 19. https://doi.org/10. 3390/ijms19082168

22. Ding JY, Kreipke CW, Speirs SL et al (2009) Hypoxia-inducible factor- $1 \alpha$ signaling in aquaporin upregulation after traumatic brain injury. Neurosci Lett 453:68-72. https://doi.org/10.1016/j.neulet. 2009.01.077

Publisher's note Springer Nature remains neutral with regard to jurisdictional claims in published maps and institutional affiliations. 